\title{
Mindfulness, Self-Compassion, Executive Functioning, and Stress: Exploring a Process Model in Adolescents
}

Received 02 June 2019

Accepted 26 August 2019

www.ijpefs.com

\section{Sarah Ullrich-French a ${ }^{*}$, Anne E. Cox a}

a Washington State University, PO Box 641410, Pullman, WA 99164, USA

*Corresponding Author Email: sullrich@wsu.edu

Abstract: The association of trait mindfulness with emotional well-being has been found to be mediated by executive functioning. However, there is little empirical evidence on this process in adolescents. Therefore, this study tested these associations using an adolescent sample participating in a physical education yoga class. This study extended previous research by also including self-compassion and state mindfulness in addition to trait mindfulness. A prospective design employed a pilot yoga curriculum in a high school physical education class. Adolescents $(N=20)$ completed assessments of trait mindfulness and self-compassion at baseline, state mindfulness experienced during yoga classes over the 12 week physical education yoga class, and six indices of executive functioning and stress at the end of the 12 weeks. Path analysis was used to test the process model found by Short with the extensions of self-compassion and state mindfulness. Self-compassion directly predicted problems with activity level impulse control and indirectly predicted stress. When self-compassion did not predict specific executive functioning indicators, state mindfulness experienced in yoga predicted stress. This study contributes preliminary evidence that suggests further research into the unique effects of trait and state mindfulness as well as self-compassion on adolescent cognitive and affective outcomes. Results support the use of contemplative practices, such as yoga, in adolescent physical education as a strategy to boost emotion regulation processes.

Key Words: State Mindfulness, Physical Education, Psychological Resilience, Affect, Yoga

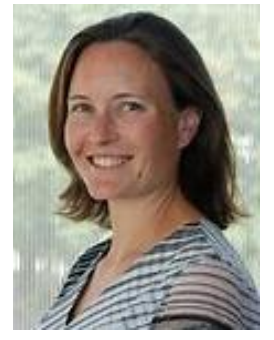

Sarah Ullrich-French is an Associate Professor of Kinesiology at Washington State University. She received her MS and PhD in Kinesiology with an emphasis on Sport and Exercise Psychology from Purdue University. Dr. Ullich-French is an Associate Editor for Sport, Exercise, and Performance Psychology. Her research examines social-contextual factors as well as intra-individual factors in motivational processes for physical activity. Currently her work focuses on the interface of mindfulness and self-compassion in body image and physical activity motivation.

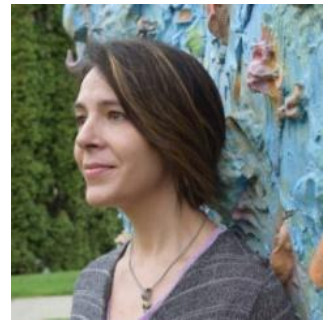

Anne Cox is an Associate Professor of Kinesiology at Washington State University. She received her $M A$ from the University of Virginia and PhD from Purdue University in the specialty of Sport and Exercise Psychology. Dr. Coxcurrently serves as the Secretary/Treasurer for the North American Society for the Psychology of Sport and Physical Activity. Her research broadly addresses key determinants of physical activity behavior. Her current area of emphasis investigates how body image variables impact physical activity motivation and behavior with an emphasis on the yoga context and the role of mindfulness and self-compassion in positive physical activity experiences. Dr. Cox has completed 200 hours of yoga teacher training and currently teaches yoga.

\section{Introduction}

Lazarus and Folkman [1] conceptualize psychological stress as an emotional response to one's appraisal of an imbalance between a stressor and one's perceived ability to cope with it. Perceived pressure, unrealistic expectations, unfavorable comparisons to others and fear of failure contribute to appraisal processes that fuel psychological stress 


\section{Sarah Ullrich-French, Anne E. Cox /2019}

for adolescents [2]. Thus understanding mechanisms that impact the appraisal process is a critical step in mitigating negative adolescent emotional experiences. Mindfulness-based school interventions have recently exploded in order to enhance adolescent cognitive and emotional well-being [3-5]. Although the emerging evidence is promising it is clear that we have much to learn about the processes explaining how mindfulness serves this purpose during adolescence [3-6].

Mindfulness is defined as present-focused attention with characteristics of nonjudgment and openness to one's experience [7, 8]. The processes underlying the effects of mindfulness on stress are not fully elucidated [e.g., 9], particularly with adolescent samples. Mindfulness research with adults demonstrates that mindfulness is associated with neurocognitive self-regulation processes of executive functions, attention, and memory [10]. Teper, Segal, \& Inzlicht [11] proposed a model suggesting that mindfulness activates self-regulation processes through the present-moment awareness and non-judgmental acceptance of emotional experiences, thus providing cues which stimulate executive functions in order to modulate emotional well-being. Thus, mindfulness may reduce a negative stress response by way of executive functions moderating the appraisal process, to apply Lazarus and Folkman's perspective. This model theoretically links mindfulness with emotional experiences, such as stress, by way of executive functioning, a neuropsychological construct representing skills involved in forming, maintaining, and shifting mental sets used in goal-directed behavior [12].

Short, Mazmanian, Oinonen, and Mushquash [13] explored a model to evaluate whether the relationship between trait mindfulness and emotional well-being was mediated by executive functioning and self-regulation using a two phase study in young adults. In phase one, self-reported dispositional mindfulness and neurocognitive performance were assessed. Four weeks later, in phase two, self-reported executive functioning, selfregulation, and emotional well-being were assessed. Results supported a model where self-reported trait mindfulness at time one directly predicted time two assessed self-reported executive functioning and selfregulation, and indirectly predicted emotional wellbeing. A recent study found inverse associations between distress and trait mindfulness and executive functioning in a cross sectional study with early adolescents [14], however, there is limited evidence of how these variables associate in adolescent populations. In addition to trait mindfulness, there is evidence that state or situational mindfulness has independent associations with emotional well-being [15-17]. However, there is no research examining trait and state mindfulness side by side as potential mechanisms for explaining stress in adolescents.

Self-compassion is another core mechanism that may help explain adolescent emotional wellbeing. Self-compassion involves a compassionate stance towards one's self in times of failure or suffering through self-kindness, a sense of common humanity, and mindfulness [18] and has received initial support for adolescents' adaptive regulation of cognitions and emotions [19]. Although there is some conceptual overlap with mindfulness, selfcompassion explains unique variance in emotional well-being beyond mindfulness in adults [20, 21]. Though self-compassion strategies have been included as part of mindfulness programming in schools (see 22, 23) we only have limited evidence linking aspects of self-compassion with adolescent stress $[19,24,25]$ and no empirical tests linking selfcompassion to adolescent executive functioning. Given the similar functions underlying mindfulness and self-compassion, executive functioning may also serve to mediate the association between selfcompassion and emotional responses. Exploring these processes is especially important given the interest in contemplative programs, such as yoga, for youth and limited empirical evidence.

The purpose of this study was to extend the model supported by Short et al. [13] to an adolescent sample and to include both trait and state mindfulness as well as self-compassion to predict stress by way of executive functioning. We tested our proposed model (see Figure 1) with a pilot study employing a 12-week yoga curriculum. 


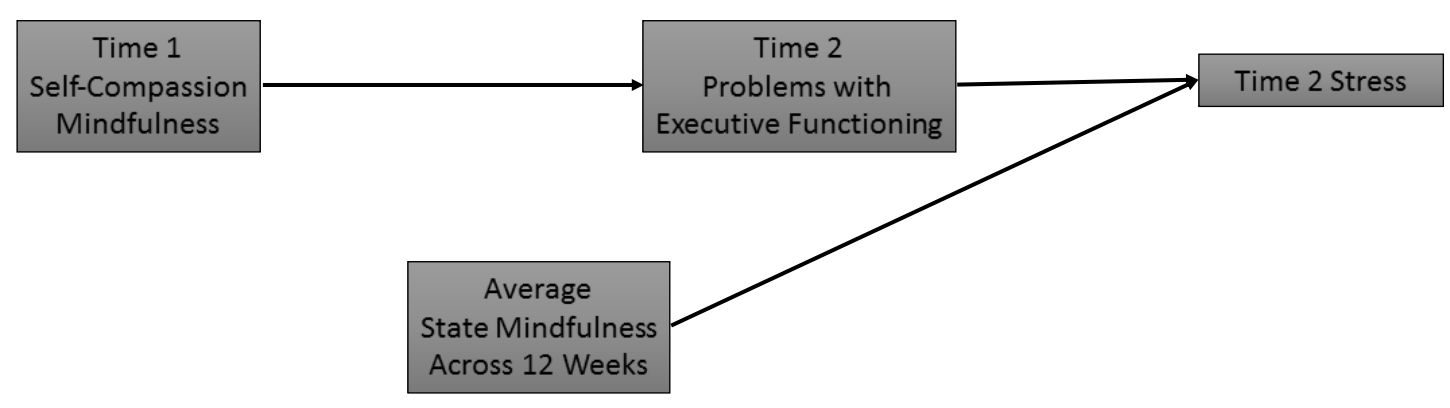

Figure 1. Hypothesized Model

This model was relevant to test in this context given that no mean changes were observed in trait level mindfulness, self-compassion, or stress ${ }^{1}$. Based on Short et al. we hypothesized that trait mindfulness and self-compassion at time one would negatively predict problems with executive functioning directly and stress indirectly at time two. Based on Brown Ryan [15], Kiken et al. [16], and Weinstein et al. [17], we hypothesized that students' average levels of state mindfulness experienced during the yoga classes across the 12 weeks would negatively predict stress directly.

\section{Methods}

\subsection{Participants and Procedures}

Participants $(N=20)$ were primarily Caucasian $(85.0 \%)$ and female $(90 \%)$ and in $10^{\text {th }}$ through $12^{\text {th }}$ grades $\left(M_{\text {age }}=16.45, S D=1.0\right)$. Twenty-three students participated in the yoga curriculum ${ }^{2}$ as part of their physical education (PE) class (one student joined late and was not included; another student had developmental difficulties and one student did not complete the final assessments). Each week of the 12-week curriculum was led by a certified yoga instructor (200-hour Anusara Yoga Teacher Training certification program) and focused on a theme (e.g., self-compassion; overcoming obstacles) that was integrated into two yoga sessions that week (1 50minute and 1 80-minute). Each yoga practice included an introduction and centering exercise, practice moving through and holding different asanas (i.e., poses) and a closing and meditation exercise.
Students in this class typically elect this class to fulfill their PE requirement, do not participation in school sports, may have low interest in traditional physical education, and are mostly female. The majority (90\%) of participants reported beginning to beginning intermediate yoga experience. Participants completed the measures of trait mindfulness and self-compassion the week before beginning a 12 week yoga program (time one) in their physical education class. State mindfulness was assessed immediately following one yoga class per week. At the end of twelve weeks, participants completed the measures of executive functioning and stress (time two).

\section{2 Measures}

Trait mindfulness was assessed using Cardaciotto, Herbert, Forman, Moitra, \& Farrow's [26] 20-item Philadelphia Mindfulness Scale. Two subscales capture trait awareness (e.g., "I am aware of what thoughts are passing through my mind") and acceptance (e.g., "There are things I try not to think about"). Responses fall on a 5-point Likert scale of never to very often, with higher scores on each scale reflecting higher levels of mindful awareness or acceptance. This measure has supporting evidence of internal consistency reliability and validity [26]. Alpha reliability was low for both trait mindfulness scales. Several items ${ }^{3}$ had low inter-item correlations. With these items removed, reliability improved for the awareness scale $(\alpha=.71)$ and the acceptance scale $(\alpha=.69)$. 


\section{Sarah Ullrich-French, Anne E. Cox /2019}

Self-compassion was assessed using Neff's [27] SelfCompassion Scale. The 26-item scale assesses three positive (self-kindness, common humanity, mindfulness) and three negative (self-judgement, isolation, over-identification) dimensions underlying self-compassion. Participants respond to how often they experience a particular response to pain and suffering (e.g., "I see my failings as part of the human condition" - common humanity; "When I'm feeling down I tend to obsess and fixate on everything that is wrong" - over-identification) using a 5 point scale ranging from almost never to almost always. A total average score was calculated where higher scores reflect higher self-compassion. Conceptual and psychometric properties of the scale are supported, including for adolescents [28, 29].

Stress was assessed using the Perceived Stress Scale [30]. The PSS items tap into the perceived level of stress experienced in the last week (modified from last month to provide a more proximal indicator of perceived stress; similar modifications are not expected to decrease validity) (e.g., "In the last week, how often have you felt difficulties were piling up so high that you could not overcome them"). The PSS contains 14 items and is scored on a 5 point scale with response options ranging from never to very often. The PSS has supporting evidence for psychometric properties [30] and use in adolescent samples (e.g., 24).

Executive functioning was assessed with the self-report form of the Delis Rating of Executive Functions (D-REF) [31], a rating scale of reported behavioral problems for 11-18 year-olds. There are 36 items asking the individual to rate the frequency of behaviors in the last 6 months on a 4-point scale ranging from seldom or never to daily. These items are used to calculate six scores or indices of executive function. Three scores represent dysfunction relative to 1) behavior ("I say things I wish I hadn't"), 2) emotion ("I try to control my anger but I just can't"), and 3) cognition ("I have a difficult time putting my thoughts down in writing"). There are also three clinically derived scores representing dysfunction of 1) attention \& working memory ("I get confused when I have two or more things to do at the same time"), 2) activity level impulse control ("I just can't help doing things that I'm told not to do"), and 3) compliance \& anger management ("I get really upset when people interfere with what I'm doing"). The six indices are presented as $\mathrm{T}$ scores, age and gender adjusted, with values of 50 representing the norm sample average. Evidence supports reliability, content, construct, and concurrent validity [31]. Internal consistency reliability information was not available for scores obtained through the DREF online interface.

State mindfulness experienced during the yoga classes was assessed immediately following class each week using the State Mindfulness Scale for Physical Activity (SMS-PA) [32]. The SMS-PA has six items each capturing mindfulness of mental (e.g., "I was aware of different emotions that arose in me") and physical objects (e.g., "I felt present in my body") during a specific experience. Responses to how much each item was experienced fall on a 5-point scale ranging from not at all to very much. All 12 items were averaged with higher scores representing higher mindfulness during the yoga class. Weekly scores were averaged across the twelve weeks to represent average state mindfulness experienced in the yoga class. Initial evidence supports the internal consistency reliability, factorial, and construct validity using adult samples [32] and with youth samples as young as 10 years-old [33]. Alpha reliability was good across assessments $\left(\alpha_{\text {range }}=.76-\right.$ $.97)$ and when averaged across time points $\left(\alpha_{\text {average }}=\right.$ .90).

\section{3 Data Analysis}

Data screening for missing values and normality was conducted and descriptive statistics were calculated. Hypothesized mediational models were tested with path models using Mplus 7. Time one scores of mindfulness (i.e., trait acceptance, and awareness) and self-compassion were entered into the model directly predicting executive functioning at time two and indirectly predicting stress at time two through executive functioning as per Short et al. [13]. Average state mindfulness during the yoga classes across the 12 weeks was also entered as a direct predictor of stress at the end of the 12 weeks (see Figure 1). Six separate models were run to test each 


\section{Sarah Ullrich-French, Anne E. Cox / 2019}

of the six indices of executive functioning. To obtain imputation was used on all constructs except $95 \%$ confidence intervals, we conducted executive functioning indices $(.01 \%$ missing but not bootstrapping specifying 5000 samples to reduce imputed as recommended [31] and state mindfulness standardized error bias and for type I error (used all available weekly state scores to create correction (see [34]). Completely standardized path average state scores, average of 8.6 of 11 scores coefficients were reported along with absolute and available). Data screening showed variables to be incremental model fit indices [35]. Given the approximately normal. See Table 1 for descriptive exploratory nature of our study and small sample statistics.

size, we report exact $p$ values, confidence intervals and effect sizes for broad interpretation as reliance on significance levels is limiting. Confidence intervals that do not cross 0.00 were interpreted as reliable. Effect sizes were interpreted as minimal (.04), moderate (.25), and strong (.64).

\section{Results}

There was negligible missing data (.02\%) and Little's MCAR test was non-significant $(\chi 2=40.34$ (47), $p=.74)$, therefore Expectation Maximization
In all the path models the acceptance and awareness trait mindfulness subscales did not significantly predict any of the indices of executive functioning. We therefore removed these subscales and proceeded to test the six mediation models with only self-compassion and state mindfulness. All models provided good fit and explained $17 \%$ to $46 \%$ of the variance in stress (see Table 2). Selfcompassion significantly predicted behavioral functioning and activity level impulse control, with a significant indirect effect to stress through activity level impulse control.

Table 1. Means, Standard Deviations, Internal Consistency Reliability, and Bivariate Correlations.

\begin{tabular}{lllllllll}
\hline & 1 & 2 & 3 & 4 & 5 & Mean & SD & Possible Range \\
\hline 1 Self Compassion & & & & & & 2.55 & 0.69 & $1-5$ \\
2 Acceptance^ & .24 & & & & & 1.57 & 0.42 & $1-5$ \\
3 Awareness^ & $.58^{*}$ & $.51^{*}$ & & & & 2.94 & 0.43 & $1-5$ \\
4 State Mindfulness $^{\wedge} .17$ & .30 & .16 & & & 2.74 & 0.56 & $1-5$ \\
5 Stress & -.29 & .29 & .08 & -.37 & & 2.34 & 0.56 & $1-5$ \\
6 BF & -.42 & .20 & -.11 & -.30 & $.46^{*}$ & 57.75 & 8.15 & $20-80$ \\
7 EMF & -.19 & .14 & -.06 & -.11 & .42 & 57.65 & 8.28 & $20-80$ \\
8 CF & -.20 & .21 & .08 & -.16 & .28 & 59.70 & 8.22 & $20-80$ \\
9 AWM & -.24 & .27 & .09 & -.06 & .24 & 59.45 & 8.31 & $20-80$ \\
$10 \mathrm{AIC}$ & -.35 & .24 & .06 & -.20 & $.64^{* *}$ & 57.61 & 6.93 & $20-80$ \\
$11 \mathrm{CAM}$ & -.14 & .17 & .02 & -.05 & .22 & 56.63 & 8.35 & $20-80$ \\
$\alpha 1$ & .93 & .69 & .71 & .90 & .81 & & & \\
\hline
\end{tabular}

Notes. ${ }^{\wedge}$ Trait mindfulness subscales; $\mathrm{BF}=$ behavior functioning, $\mathrm{EMF}=$ emotional functioning, $\mathrm{CF}=$ cognitive functioning, AWM = attention \& working memory, AIC = activity level \& impulse control, CAM = compliance $\&$ anger management. ${ }^{*} \mathrm{p}<.05^{* *} \mathrm{p}<.01$. 


\section{Sarah Ullrich-French, Anne E. Cox /2019}

Table 2. Mediation Path Analysis Result

\begin{tabular}{|c|c|c|c|c|c|c|c|c|c|c|c|}
\hline Mediator & Direct Effects & & & $\begin{array}{l}\text { Indirect } \\
\text { Effect }\end{array}$ & Mode & & & & & $R^{2}$ & \\
\hline $\begin{array}{l}\text { Executive } \\
\text { Functioning } \\
\text { Indices }\end{array}$ & $\begin{array}{l}\text { self- } \\
\text { compassion } \\
\text { to mediator }\end{array}$ & $\begin{array}{l}\text { mediator to } \\
\text { stress }\end{array}$ & $\begin{array}{l}\text { state } \\
\text { mindfulness } \\
\text { to stress }\end{array}$ & $\begin{array}{l}\text { self- } \\
\text { compassion }\end{array}$ & $\chi^{2}(2)$ & $p$ & RMSEA & SRMR & CFI & Mediator & Stress \\
\hline Behavioral & $\begin{array}{l}-.42, p=.02 \\
(-.72 \text { to }-.12)\end{array}$ & $\begin{array}{l}.39, \quad p=.09 \\
(.01 \text { to } .76)\end{array}$ & $\begin{array}{l}-.27, p=.19 \\
(-.59 \text { to } .06)\end{array}$ & $\begin{array}{l}-.16, p=.17 \\
(-.36 \text { to } .03)\end{array}$ & 1.59 & .45 & .00 & .08 & 1.00 & .18 & .23 \\
\hline Emotional & $\begin{array}{l}-.19, p=.28 \\
(-.48 \text { to } .09)\end{array}$ & $\begin{array}{l}39, p=.09 \\
(.01 \text { to } .77)\end{array}$ & $\begin{array}{l}-.34, p=.04 \\
(-.60 \text { to- } .07)\end{array}$ & $\begin{array}{l}-.08, p=.34 \\
(-.20 \text { to } .06)\end{array}$ & 0.87 & .65 & .00 & .05 & 1.00 & .04 & .27 \\
\hline Cognitive & $\begin{array}{l}-.20, p=.39 \\
(-.56 \text { to } .17)\end{array}$ & $\begin{array}{l}.23, p=.32 \\
(-.16 \text { to.62) }\end{array}$ & $\begin{array}{l}-.34, p=.05 \\
(-.62 \text { to }-.06)\end{array}$ & $\begin{array}{l}-.05, \quad p=.58 \\
\text { (-.18 to } .09)\end{array}$ & 1.29 & .53 & .00 & .06 & 1.00 & .04 & .17 \\
\hline AWM & $\begin{array}{l}-.24, p=.25 \\
(-.59 \text { to } .10)\end{array}$ & $\begin{array}{l}.22, \quad p=.26 \\
(.10 \text { to } .55)\end{array}$ & $\begin{array}{l}-.36, p=.03 \\
(-.63 \text { to }-.09)\end{array}$ & $\begin{array}{l}-.05, p=.46 \\
(-.18 \text { to } .07)\end{array}$ & 0.83 & .66 & .00 & .05 & 1.00 & .06 & .19 \\
\hline AIC & $\begin{array}{l}-.43, p=.02 \\
(-.74 \text { to }-.13)\end{array}$ & $\begin{array}{l}.64, p=.00 \\
(.31 \text { to } .96)\end{array}$ & $\begin{array}{l}-.19, p=.30 \\
(-.50 \text { to } .11)\end{array}$ & $\begin{array}{l}-.28, p=.05 \\
(-.50 \text { to }-.05)\end{array}$ & 1.44 & .49 & .00 & .10 & 1.00 & .19 & .46 \\
\hline CAM & $\begin{array}{l}-.14, p=.52 \\
(-.48 \text { to } .21)\end{array}$ & $\begin{array}{l}.20, p=.38 \\
(-.19 \text { to.59) }\end{array}$ & $\begin{array}{l}-.36, p=.04 \\
(-.64 \text { to }-.09)\end{array}$ & $\begin{array}{l}-.03, p=.67 \\
(-.14 \text { to } .08)\end{array}$ & 1.05 & .59 & .00 & .05 & 1.00 & .02 & .18 \\
\hline
\end{tabular}

Notes. AWM = attention \& working memory, AIC = activity level $\&$ impulse control, $\mathrm{CAM}=$ compliance $\&$ anger management. 


\section{Sarah Ullrich-French, Anne E. Cox /2019}

All paths predicting executive functioning were negative. These results reflect minimal to moderate effect sizes $\left(R^{2}=.18, .19\right)$.The average experience of state mindfulness during the 12 weeks of yoga classes predicted stress in all models except for behavioral functioning and activity level impulse control, demonstrating a robust negative association with stress even with executive functioning included in the model. The effect sizes predicting stress were moderate $\left(R^{2}=.17-.46\right)$.

\section{Discussion}

This study extended the literature with an initial exploration of state and trait mindfulness as well as self-compassion in predicting adolescent executive functioning and stress. Contemplative interventions with youth are popular, yet we know relatively little about the processes underpinning mindfulness and compassion. Preliminary support for the hypothesized role of executive functioning in explaining the relationship between self-compassion and stress was found in the prediction of behavioral functioning and activity level impulse control, with a significant indirect effect to stress through activity level impulse control. These results are consistent with Short et al [13] who also supported a model with self-reported dysfunction in executive functioning mediating the association of trait level psychological resilience (i.e., mindfulness) and negative emotional well-being (i.e., negative affect). Emerging empirical evidence linking self-compassion with the emotional well-being of adolescents [19, 24] coupled with the moderate effect sizes in our results indicate that self-compassion as a resilience factor in adolescents is worthy of more rigorous exploration.

Impulse control has been linked with managing emotional responses with short-term emotional relief over longer-term goal directed emotional well-being [36]. Self-compassion may reduce problems with impulse control and associated behavior regulation problems by allowing one to experience suffering in a gentle way and reducing the need to avoid negative emotional experiences through behavioral responses, such as speaking before thinking. Even with a limited sample size, we were able to detect these associations, with minimal
Both trait, or dispositional levels of psychological variables, such as self-compassion, and state level experiences may be important to consider when examining the effects of contemplative interventions and is supported by work with adults demonstrating the independent effects of state and trait mindfulness (e.g., 15). Thus, interventions that foster state mindfulness and self-compassion may reduce stress responses, regardless of students' dispositional tendencies in mindfulness. Even in cases where students' dispositional tendencies do not change, as was the case in this study, these statelevel experiences may effect change in well-being. These results reflect recent systematic reviews of the mindfulness literature which suggest that more than half of mindfulness interventions do not demonstrate significant self-reported mindfulness change [37] and that assessment of mindfulness practice is a significant predictor of emotional well-being $[38,17]$. Further work using more rigorous experimental research designs could shed more light on the relative importance of changes in dispositions versus state experiences during contemplative-based interventions. Further, although we aligned our model with the procedures of Short et al [13], true mediation should be tested with executive functioning assessed prior to stress [39].

The trait mindfulness subscales (acceptance, awareness) did not significantly predict any of the indices of executive functioning. It is unclear if this was due to marginal reliability of these scales which have limited use with adolescent samples and/or low power. The Philadelphia Mindfulness Scale was used in this study as it conceptually aligned with the suggested mechanisms for the effectiveness of mindfulness through scales of both acceptance and awareness [11], however, validation with adolescent samples is needed. Research with adults has sometimes demonstrated the stronger role of selfcompassion relative to mindfulness when predicting emotional well-being [20, 21], which could explain this finding. Further research is necessary to test the 


\section{Sarah Ullrich-French, Anne E. Cox /2019}

hypothesized model with trait mindfulness, executive functioning and stress in larger, more representative adolescent samples.

Our results should be considered as preliminary and viewed within limitations of sample and design. Future studies can build from this preliminary evidence by examining larger, more diverse samples with the use of more rigorous experimental research designs to detail the unique effects of trait mindfulness, self-compassion, and state mindfulness in adolescent emotion regulation processes. The representation of executive functioning also deserves attention as there are a broad array of approaches to study executive functioning. Further research exploring more rigorous assessment of executive functioning is needed as well as both behavioral and self-reported assessments [40].

\section{Conclusions}

This study explored the association of mindfulness and self-compassion with executive functioning and stress in adolescents. By including self-compassion in this study we extended Short et al.'s findings to a construct that is both conceptually and empirically distinct from mindfulness. There is also recent interest in the role of mindful states, for example in reducing rumination, supporting further exploration of state mindfulness. This study also provides promising evidence supporting further examination of the role of state mindfulness and selfcompassion in reducing adolescent stress, areas of study with minimal empirical research evidence, but high popularity and enthusiasm for implementation.

\section{References}

[1] R.S. Lazarus, S. Folkman, (1984) Stress, appraisal, and coping, Springer Publishing Company, New York, NY.

[2] T.A. Murberg, E. Bru, School-related stress and psychosomatic symptoms among Norwegian adolescents, School Psychology International, 25 (2004) 317-332.
[3] Kechter, D.S. Black, (2018) Mindfulness meditation interventions with children and adolescents: Research findings and implications for social work practice In Integrative Body-Mind-Spirit Social Work An Empirically Based Approach to Assessment and Treatment (2nd Edition), Oxford University Press, New York, NY.

[4] Mak, K. Whittingham, R. Cunnington, R.N. Boyd, Efficacy of mindfulness-based interventions for attention and executive function in children and adolescents - a systematic review, Mindfulness, 9 (2018) 5978.

[5] C. Zenner, S. Herrnleben-Kurz, \& H. Walach, Mindfulness-based interventions in schools: A systematic review and meta-analysis, Frontiers in Psychology, 5 (2014) 603.

[6] R.J. Davidson, J. Dunne, J.S. Eccles, A. Engle, M. Greenberg, M., P. Jennings, D. Vago, Contemplative practices and mental training: Prospects for American education. Child Development Perspectives, 6 (2012) 146-153.

[7] Scott R. Bishop Mark Lau Shauna Shapiro Linda Carlson Nicole D. Anderson James Carmody Zindel V. Segal Susan Abbey Michael Speca Drew Velting Gerald Devins Mindfulness: A proposed operational definition. Clinical Psychology: Science and Practice, 11 (2006) 230-241.

[8] J. Kabat-Zinn (2005) Coming to our senses: Healing ourselves and the world through mindfulness, Hyperion, New York, NY.

[9] B. Isbel, \& D. Mahar, Cognitive mechanisms of mindfulness: A test of current models, Consciousness and Cognition, 38 (2015) 5059.

[10] A. Chiesa, R. Calati, \& A. Serretti, Does mindfulness training improve cognitive abilities? A systematic review of neuropsychological findings, Clinical Psychology Review, 31 (2011) 449-464.

[11] R. Teper, Z.V. Segal, \& M. Inzlicht, Inside the mindful mind how mindfulness enhances emotion regulation through improvements in executive control, Current Directions in Psychological Science, 22 (2013) 449-454. 


\section{Sarah Ullrich-French, Anne E. Cox /2019}

[12] Y. Suchy, Executive functioning: Overview, [21] assessment, and research issues for nonneuropsychologists, Annals of Behavioral Medicine, 37 (2009) 106-116.

[13] M.M. Short, D. Mazmanian, K. Oinonen, \& C.J. Mushquash, Executive function and selfregulation mediate dispositional mindfulness [22] and well-being, Personality and Individual Differences, 93 (2016) 97-103.

[14] A. Kechter, D.S. Black, N.R. Riggs, C.M. Warren, A. Ritt-Olson, C-P. Chou, \& M.A. Pentz, Factors in the Perceived Stress Scale Differentially Associate with Mindfulness Disposition and [23] Executive Function among Early Adolescents, Journal of Child and Family Studies, 28 (2019) 814-821.

[15] K.W. Brown, \& R.M. Ryan, The benefits of being present: The role of mindfulness in [24] psychological well-being, Journal of Personality and Social Psychology, 84 (2003) 822-848.

[16] L.G. Kirken, E.L. Garland, K. Bluth, O.S. Palsson, \& S.A. Gaylord, From a state to a [25] trait: Trajectories of state mindfulness in meditation during intervention predicts changes in trait mindfulness, Personality and Individual Differences, 81 (2015) 41-46.

[17] N. Weinstein, K.W. Brown, \& R.M. Ryan, A multi-method examination of the effects of mindfulness on stress attribution, coping, and emotional well-being, Journal of Research in Personality, 43 (2009) 374-385.

[18] K.D. Neff, Self-compassion: An alternative conceptualization of a healthy attitude toward oneself. Self and Identity, 2 (2003) 85- [28] 102.

[19] K. Bluth, \& P.W. Blanthon, The influence of self-compassion on emotional well-being among early and older adolescent males and females, Journal of Positive Psychology, 10 (2015) 219-230.

[20] R.A. Baer, E.L.B. Lykins, \& J.R. Peters, Mindfulness and self-compassion as predictors of psychological well-being in long-term mediators and matched nonmediators, The Journal of Positive Psychology, 7 (2012) 230-238.
N.T. Van Dam, S.C. Sheppard, J.P. Forsyth, \& M. Earleywine, Self-compassion is a better predictor than mindfulness of symptom severity and quality of life in mixed anxiety and depression, Journal of Anxiety Disorders, 25 (2011) 123-130.

J. Meiklejohn, C. Phillips, M. L. Freedman, M. L. Griffin, G. Biegel, A. Roach, A. Saltzman, Integrating mindfulness training into $\mathrm{K}-12$ education: Fostering the resilience of teachers and students, Mindfulness, 3 (2012) 291-307.

R. W. Roeser, \& C. Pinela, Mindfulness and compassion training in adolescence: $\mathrm{A}$ developmental contemplative science perspective, New Directions for Youth Development, 142 (2014) 9-30.

K. Bluth, P.N.E. Roberson, S.A. Gaylord, K.R. Faurot, K.M. Grewen, S. Arzon, \& S.S. Girdler, Does self-compassion protect adolescents from stress?, Journal of Child and Family Studies, 25 (2016) 1098-1109.

K. D. Neff, \& P. McGeehee, Self-compassion and psychological resilience among adolescents and young adults, Self and Identity, 9 (2010) 225-240.

[26] L. Cardaciotto, J.D. Herbert, E.M. Forman, E. Moitra, \& V. Farrow, The assessment of present-moment awareness and acceptance: The Philadelphia Mindfulness Scale, Assessment, 15 (2008) 204-223.

[27] K. D. Neff, Development and validation of a scale to measure self-compassion, Self and Identity, 2 (2003) 223-250.

M. Cunha, A. Zavier, \& P. Castilho, Understanding self-compassion in adolescents: Validation study of the selfcompassion scale, Personality and Individual Differences, 93 (2016) 56-62.

K. D. Neff, I. Toth-Kiraly, L.M., Yarnell, K. Arimitsu, P. Castilho, N. Ghorbani \& M. Mantzios, Examining the factor structure of the Self-Compassion Scale in 20 diverse samples: Support for use of a total score and six subscale scores, Mindfulness, 31 (2019) 27-45. 


\section{Sarah Ullrich-French, Anne E. Cox /2019}

[30] S. Cohen, T. Kamarck, \& R. Mermelstein, A global measure of perceived stress, Journal of Health and Social Behavior, 24 (1983) 385- [36] 396.

[31] D.C. Delis, (2012) Delis rating of executive functioning, Pearson, Bloomington, MN

[32] C.J. Ferguson, An effect size primer: A guide for clinicians and researchers, Professional Psychology: Research and Practice, 40 (2012) 532-538.

[33] A.Cox, S. Ullrich-French, \& B. French, Validity [38] evidence for state mindfulness scale scores in a physical activity context, Measurement in Physical Education and Exercise Science, 20 (2016) 38-49.

[34] S. Ullrich-French, A.E. Cox, A.N. Cole, B.R. [39] Cooper, \& C. Gotch, Initial validity evidence for the State Mindfulness Scale for Physical Activity with youth, Measurement in Physical Education and Exercise Science, 21 (2017) 177-189.

[35] A.F. Hayes, \& K.J. Preacher, Statistical [40] mediation analysis with a multicategorical independent variable, British Journal of
Mathematical and Statistical Psychology, 67 (2014) 451- 470.

R.B. Kline, (2005) Principles and Practice of Structural Equation Modeling (2 ${ }^{\text {nd }}$ Edition), The Guilford Press, New York.

D.M. Tice, E. Bratslavsky, \& R.F. Baumeister, Emotional distress regulation takes precedence over impulse control: If You Feel Bad, Do It!, Journal of Personality and Social Psychology, 80 (2001) 53-67.

E. Visted, J. Völlestad, M.B. Nielsen, \& G.H. Nielsen, The impact of group-based mindfulness training on self-reported mindfulness: A systematic review and metaanalysis, Mindfulness, 6 (2015) 501-522.

W. Kuyken, K. Weare, O. Ukoumunne, R. Vicary, N. Motton, R. Burnette, F. Huppert, Effectiveness of the Mindfulness in Schools Programme: Non-randomised controlled feasibility study, British Journal of Psychiatry, 203, (2013) 126-131.

D.P. MacKinnon, A.J. Fairchild, \& M.S. Fritz, Mediation analysis, Annual Review of Psychology, 58 (2007) 593-614.

\section{Funding}

This study was not funded by any grant

\section{Acknowledgements}

We would like to thank Amy Cole and Peter Anderson for their assistance with collecting data for this study. We would also like to thank Kristine Petterson for her contributions to the conceptualization of, and teaching, the yoga class and Tara Briggs for her collaboration with us in this project through the Pullman High School physical education program.

\section{Conflict of interest}

None of the authors have any conflicts of interest to declare.

\section{About The License}

\section{()ㅜ)}

Attribution 4.0 International (CC BY 4.0)
The text of this article is licensed under a Creative Commons Attribution 4.0 International License 